\title{
Aportaciones al conocimiento de la distribución de varias orquídeas silvestres en la provincia de Palencia (N de España)
}

\author{
J. Antonio Ruiz de Gopegui ${ }^{1}$, Alberto Rodríguez ${ }^{1}$, Enrique Álvarez², Mónica Vélez ${ }^{3}$ y Patricio Bariego ${ }^{4}$
}

Resumen: Ruiz de Gopegui, J. A.; Rodríguez, A.; Álvarez, E.; Vélez, M. \& Bariego, P. 2012. Aportaciones al conocimiento de la distribución de varias orquídeas silvestres en la provincia de Palencia (N de España). Bot. Comp. 36: 97-103.

Se aporta información sobre 18 taxones de la familia Orquidáceas pertenecientes a un total de 11 géneros, presentes en la provincia de Palencia. De ellas, 9 suponen primeras citas provinciales y 9 están incluidas en listados de flora protegida.

Palabras clave: orquídeas, Orchidaceae, flora amenazada, Palencia, España.

Abstract: Ruiz de Gopegui, J. A.; Rodríguez, A.; Álvarez, E.; Vélez, M. \& Bariego, P. 2012. Contributions to the knowledge of the distribution of some wild orchids in the province of Palencia (N Spain). Bot. Comp. 36: 97-103.

Information about eighteen taxa of Orchidaceae family belonging to 11 genera, all of them present in Palencia province, is given. Nine of them are new provincial records and 9 are includes into an endangered flora list.

Key words: Orchids, Orchidaceae, endangered flora, Palencia, Spain.

\section{INTRODUCCIÓN}

En los últimos años se ha realizado una intensa prospección florística de las diferentes comarcas palentinas al objeto de realizar un atlas de la orquidoflora provincial. Como avance de dicho trabajo se aporta información sobre la distribución de varias orquídeas que no habían sido citadas en el ámbito provincial o que han resultado ser raras 0 escasas en esta provincia, teniendo algunas de ellas la consideración de amenazadas en los listados de flora protegida 0 de interés para a conservación de ámbito regional o nacional.

Para cada taxón, ordenados alfabéticamente, se detallan los siguientes datos: municipio, localidad y topónimo, cuadrícula UTM de $1 \times 1 \mathrm{~km}$, altitud, hábitat, fecha de recolección o fotografía, colector/es y número de recolección o referencia de la existencia de fotografía testigo y, en su caso, registro del herbario en el que se han depo- sitado los pliegos testigo (Herbarios LEB-Jaime Andrés Rodríguez de la Universidad de León, MA del Real Jardín Botánico de Madrid o SALA de la Universidad de Salamanca).

Se comentan las citas previas en la provincia, indicadas con el simbolo *, así como otros datos de interés sobre su corología, rareza e inclusión en catálogos 0 mención en documentos técnicos de flora protegida 0 amenazada. También se ha incluido un anexo fotográfico para documentar alguna de las especies más conflictivas.

\section{RESULTADOS}

*Cephalanthera longifolia (L.) Fritsch

ESPAÑA: PalENCIa: Antigüedad, cabecera de arroyo de Valdefranco, 30TVM0939, 890 m, quejigar maduro, 26-IV-2011, A. Rodrí-

\footnotetext{
${ }^{1}$ GEMPA (Grupo de Estudios de Montaña - Palencia). C/ La Pontona, n ${ }^{0}$ 10. 34846 Rebanal de los Caballeros, Palencia, España. gopeguiinsitu@yahoo.es

${ }^{2}$ C/ Vitoria, $n^{0} 50$, esc. $3^{\text {a }}, 3^{0}$ B. 09004 Burgos, España. qqalvgom@gmail.com

${ }^{3} \mathrm{C} /$ Independencia $n^{\circ} 4,1^{\circ}$ A. 34003 Palencia, España. movelred@gmail.com

${ }^{4}$ C/ Candelaria Ruiz del Árbol, 37, $4^{\circ}$ A. 49017 Zamora. pbariego@hotmail.es

Recibido: 20 marzo 2012. Aceptado: 27 marzo 2012.
} 
guez, LEB 105917; Vertavillo, Valdileja, 30TUM9329, 870 m, margas básicas, quejigar, 02-V-2011, P. Bariego PB 3674 \& E. Álvarez, LEB 105635; Castrejón de la Peña, Traspeña de la Peña, 30TUN5437, 1117 m, pinar de repoblación, 18-V-2011, Y. Ruiz \& A. Ruiz de Gopegui, LEB 105773.

En la provincia de Palencia sólo se conocía la indicación provincial, entre paréntesis, de Flora iberica (Alarcón \& Aedo 2005), probablemente basada en el trabajo divulgativo de Oria de Rueda et al. (1996: 157) a tenor de lo señalado en el trabajo previo a la monografía (Alarcón \& Aedo 2002: 238). Mucho más escasa y de distribución más restringida en la provincia que su congénere $C$. $d a$ masonium (Mill.) Druce, resulta localmente abundante en los quejigares mejor conservados de varios municipios de la comarca del Cerrato y también ha sido localizada puntualmente en la montaña palentina.

\section{*Dactylorhiza fuchsii (Druce) Soó}

ESPAÑA: Palencia: La Pernía, Lores, 30TUM7261, $1360 \mathrm{~m}$, prado higroturboso, 18-VI-2011, A. Ruiz de Gopegui, LEB 105392; Ibidem, 30TUN7361, $1327 \mathrm{~m}$, prado higroturboso, 18-VI-2011, A. Ruiz de Gopegui (Fig. 1).

Novedad provincial de la que únicamente se han encontrado dos poblaciones, cercanas entre sí, en zonas de higroturbosas de la montaña palentina. La minuciosa revisión de las localidades cantábricas realizadas por Argüelles et al. (2005), ya anticipaba su más que probable presencia en la montaña palentina. En la provincia de Palencia, tal y como sucede en la provincia de Burgos (Alejandre et al. 2006: 813), es una planta de distribución más restringida y mucho más escasa que su congénere $D$. maculata (L.) Soó.

\section{Epipactis palustris (L.) Crantz}

ESPAÑA: Palencia: Cervera de Pisuerga, Vado, 30TUN7945, $1000 \mathrm{~m}$, pradera encharcada, 25-VII-2010, A. Ruiz de Gopegui, LEB 104194; Saldaña, 30TUN5611, 950 m, pastizal rezumante, 18-V-2011, P. Bariego \& E. Álvarez; Cervera de Pisuerga, Vañes, 30TUN7755, $1043 \mathrm{~m}$, pasto higrófilo en orla de hayedo-robledal, 12-VII-2011, $A$. Ruiz de Gopegui, LEB 106062; Cervera de Pisuerga, Vallespinoso de Cervera, 30TUN7755, 950 m, cuneta encharcada, 19-VII-2011, A. Ruiz de Gopegui, LEB 104029.

Nuevas localidades a añadir a las previamente conocidas en la montaña palentina (Aedo et al. 2000: 100) y en la vega del río Carrión (Leroy \& Lainz 1954: 123), que han podido servir para la indicación provincial entre pa-

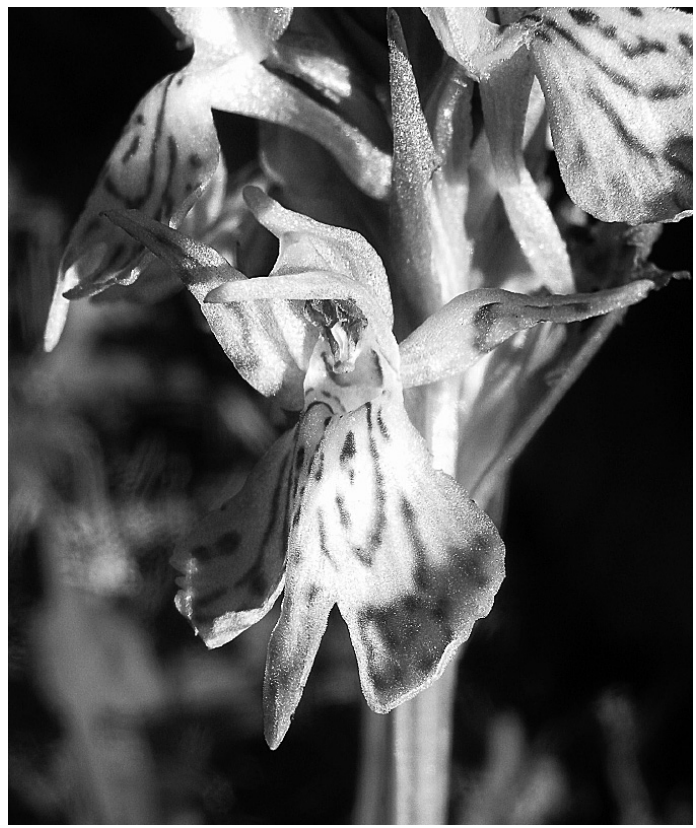

Fig. 1- Dactylorhiza fuchsii (Druce) Soó.

réntesis de Crespo (2005: 28) en Flora iberica. Consideramos que es una planta poco frecuente en la provincia, aunque podrían localizarse nuevas poblaciones en los páramos de la mitad norte provincial donde salpican el paisaje los enclaves propicios para esta especie tales como pequeñas turberas, fuentes y taludes rezumantes básicos. Se encuentra incluida en el catálogo de flora protegida de Castilla y León (Anon. 2007), así como en el listado de flora cantábrica de interés (Llamas et al. 2007).

\section{Epipactis rhodanensis Gévaudan \& Robatsch}

ESPAÑA: PaLeNCIa: Villamediana, 30TUM8448, $840 \mathrm{~m}$, bosque de ribera, 8-VI-2011, E. Álvarez, SALA 142386.

Añadimos una nueva localidad a las menciones previas de Benito \& Tabuenca (2000: 107; sub Epipactis campeadorii P. Delforge) y de Benito \& Hermosilla (1998: 106; sub Epipactis hispanica Benito Ayuso \& Hermosi1la). A juzgar por las últimas aportaciones a su distribución en la cuenca del Duero (Alejandre et al. 2006: 816; Bariego \& Gastón 2005: 153) entendemos que no debería ser una planta rara en las choperas de los principales ríos de la provincia y, en particular, en el Carrión y el Pisuerga. Incluida en el listado de flora cantábrica de interés (Llamas et al. 2007). 


\section{*Limodorum trabutianum Batt.}

ESPAÑA: Palencia: Alar del Rey, 30TUN9329, 910 m, 23-VI2011, quejigar, A. Rodríguez, LEB 106050; Pomar de Valdivia, Villaescusa de las Torres, 30TUN9634, 970 m, 24-VI-2011, quejigar en ladera caliza, A. Rodríguez, LEB 105935.

Nueva para la provincia, su distribución provincial se restringe por el momento a los quejigares calizos de la comarca de las Loras, aunque es posible que sea más amplia ya que puede haber pasado desapercibida en muchas 10calidades al convivir con frecuencia con su congénere $L$. abortivum (L.) Sw., más frecuente en la provincia. Estas localidades contribuyen a ampliar el conocimiento de su distribución en el norte peninsular, donde parece ser una planta rara, sumándose a los recogidos por Alejandre et al. (2006: 819) para Burgos y los aportados por Aedo et al. (2000: 100; 2001: 39) para Cantabria. Incluida en el listado de flora cantábrica de interés (Llamas et al. 2007) (Fig. 2).

\section{Listera ovata (L.) R. Br.}

ESPAÑA: PaLENCIA: Brañosera, Salcedillo, 30TUN9455, 1330 m, brezal higrófilo, 3-VII-2010, A. Rodríguez, LEB 104344; Cervera

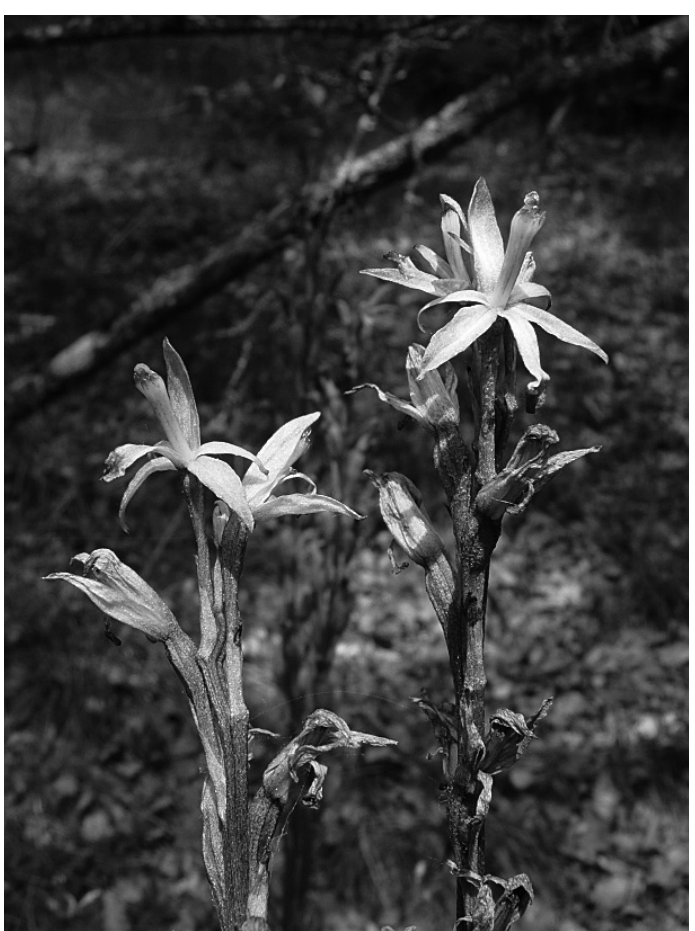

Fig. 2-Limodorum trabutianum Batt. de Pisuerga, Barcenilla, 30TUN8342, $1067 \mathrm{~m}$, interior de quejigar, 24VI-2011, Y. Ruiz \& A. Ruiz de Gopegui, LEB 106064.

Citada anteriormente en la provincia por Aedo et al. (1994: 94), aparece de forma dispersa, aunque localmente abundante, en praderas y matorrales acidófilos del tercio norte provincial. Incluida en el listado de flora cantábrica de interés (Llamas et al. 2007).

\section{${ }^{*}$ Neotinea maculata (Desf.) Stearn}

ESPAÑA: Palencia: Alar del Rey, Barrio de Santa María, 30TUN9229, $1030 \mathrm{~m}, 5$-V-2011, pasto pedregoso en hondonada de páramo calizo, A. Rodriguez, LEB 105927; Pomar de Valdivia, Revilla de Pomar, 30TVN0734, $1160 \mathrm{~m}$, hondonada en pasto de páramo calizo, 6-V-2011, A. Rodriguez, LEB 105932

Aunque resulta frecuente en gran parte de la Península Ibérica (López 2005: 112), hasta el momento no había sido citada en la provincia Palencia. Orquídea, un tanto críptica, que sólo ha sido encontrada, de forma muy localizada, en la comarca de las Loras.

\section{*Ophrys fusca subsp. dyris (Maire) Soó}

ESPAÑA: PaleNCIA: Arconada, Las Heras, 30TUM7687, $826 \mathrm{~m}$, pastizal de diente, 19-IV-2011, A. Ruiz de Gopegui, LEB 105399; San Mamés de Campos, 30TUM7191, $810 \mathrm{~m}$, pastizal de diente, 19-IV2011, A. Ruiz de Gopegui, RdeG 533; Pomar de Valdivia, Cezura, talud arenoso, 30TVN0440, $915 \mathrm{~m}, 30-\mathrm{IV}-2011$, A. Ruiz de Gopegui \& L. García, RdeG 532.

Aportamos varias localidades para este taxón que, a diferencia de la subsp. fusca que es relativamente frecuente en el norte de la provincia (Oria de Rueda et al. 1996: 264; Ruiz de Gopegui et al. 2010: 153), no había sido citado con anterioridad en Palencia. En las poblaciones palentinas del taxón conviven frecuentemente ambas subespecies y algunas formas intermedias referibles a $O$. vasconica (0. Danesch \& E. Danesch) P. Delforge, circunstancia frecuente en el N de la Península (Aldasoro \& Saez 2005: 180). La subsp. dyris está incluida en el listado de flora cantábrica de interés (Llamas et al. 2007).

\section{*Ophrys fusca subsp. bilunulata (Risso)}

Aldasoro \& L. Saez

ESPaÑA: Palencia: Aguilar de Campoo, Las Tuerces, 30TVN0132, 1037 m, 5-V-2011, A. Ruiz de Gopegui, LEB 105394. 
Al igual que la anterior subespecie, no había sido citada previamente en la provincia, aunque, tal y como señalan Alejandre et al. (2006: 821) para el ámbito burgalés, alguna de las referencias previas a $O$. fusca Link. en Oria de Rueda et al. (1996: 264) podrían corresponder a alguno de estos taxones. Orquídea bien distinguible de las otras subespecies por su labelo de márgenes aplanados y reborde amarillo neto en los bordes, de la que solo se ha localizado una pequeña población en la comarca de las Loras en el norte de la provincia (Fig. 3).

\section{Ophrys insectifera L. subsp. insectifera}

ESPAÑA: PaLENCIa: Castrillo de Don Juan, pr. arroyo de Valdefranco, 30TVM0737, $909 \mathrm{~m}$, encinar con quejigo, calizas, 10-VI-2007, L. Delgado \& M. Santos Vicente, MS-874.

Aportamos una nueva localidad en la comarca del Cerrato, a añadir a la primera mención provincial en las loras del norte de la provincia (Ruiz de Gopegui et al. 2010: 133), que amplía notablemente su área de distribución palentina.

\section{Orchis coriophora $\mathrm{L}$.}

ESPAÑa: Palencia: Brañosera, Valberzoso, 30TUN9853, 1100 m, pastizal de diente, 22-VI-2010, A. Ruiz de Gopegui, LEB 104198;

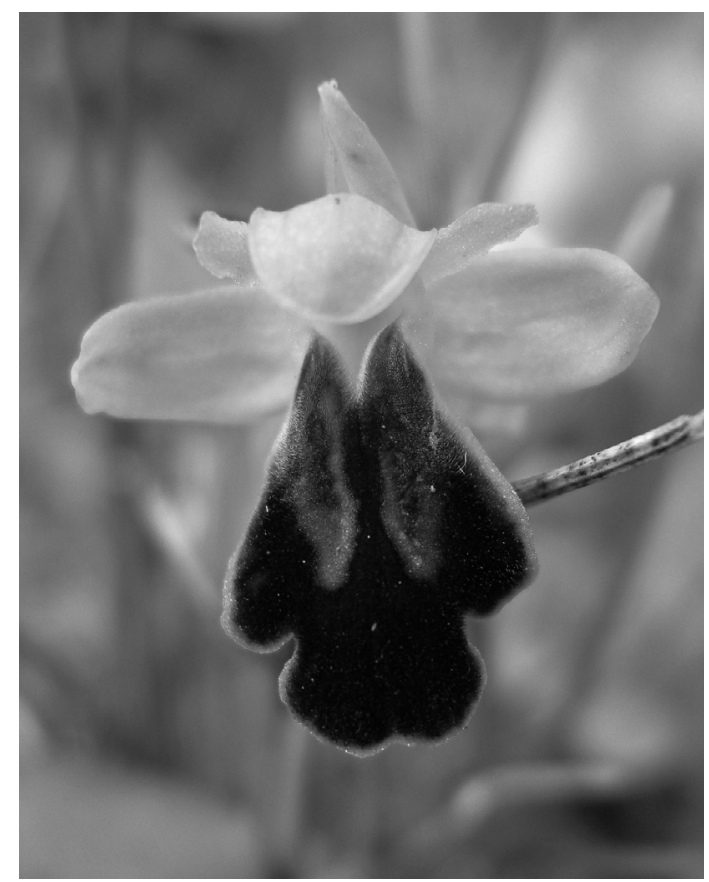

Fig. 3-Ophrys fusca subsp. bilunulata (Risso) Aldasoro \& L. Saez.
Brañosera, 30TUN9354, 1310 m, pastizal de diente, 24-VI-2010, $Y$. Ruiz \& A. Ruiz de Gopegui, LEB 104044; Brañosera, Salcedillo, 30TUN9455, $1330 \mathrm{~m}$, pasto edafohigrófilo con facies de brezal, 03VII-2010, A. Rodríguez, LEB 104630; Amusco, 30TUM7570, 770 m, pradera higroturbosa, 29-VI-2010, A. Rodríguez, LEB104131; Salinas de Pisuerga, Monasterio, 1090 m, pastizal, 30TUN8848, 28-V-2011, E. Álvarez, M. Velez \& J. Cuesta; Cervera de Pisuerga, Valsadornín, 30TUN7848, orla de robledal, 1080 m, 9-VI-2011, Y. Ruiz \& A. Ruiz de Gopegui, LEB 105400; Aguilar de Campoo, Cordovilla de Aguilar, 30TUN9948, 1016 m, pastizal de diente, 11-VI-2011, A. Ruiz de Gopegui \& L. García, LEB 104196.

Completamos la distribución provincial de esta orquídea, cuyas referencias previas se limitan a la vaga mención de Gandoger (1898: 599) en su listado de herborizaciones en Cervera de Pisuerga, que pudo servir de base para la indicación provincial entre paréntesis de Flora iberica (Aedo 2005: 123), dibujando un área que alcanza desde el norte provincial a la comarca de Tierra de Campos, donde se conoce una única población muy reducida.

\section{Orchis papilonacea $\mathrm{L}$.}

ESPAÑA: Palencia: Cervera de Pisuerga, Rabanal de los Caballeros, 30TUN7950, 1000 m, pastizal, 26-IV-2004, A. Ruiz de Gopegui, MA 749635; Astudillo, 30TUM9163, $840 \mathrm{~m}$, pastizal en paramera caliza, 23-V-2007, E. Álvarez et al.; La Pernía, Lores, 30TUN7461, $1250 \mathrm{~m}$, borde de prado de siega, 29-V-2008, E. Álvarez \& A. Ruiz de Gopegui; Cervera de Pisuerga, Herreruela de Castillería, 30TUN8554, 1260 m, 8-V-2011, A. Ruiz de Gopegui, RdeG 546; Bustillo del Páramo, 30TUM5592, $850 \mathrm{~m}, 14-\mathrm{V}-2011$, pradera en borde de melojar, $A$. Rodriguez, LEB 105912.

Citada anteriormente en la Montaña Palentina por Aedo et al. (1993: 368) y Ruiz de Gopegui et al. (2010: 154), ampliamos ahora su distribución a los páramos del $\mathrm{N}$ y el SE provincial. A pesar de su extensa distribución provincial, no deja de ser una planta rara ya que sus poblaciones suelen tener un reducido número de individuos. Está recogida en el catálogo de flora protegida de Castilla y León (Anon. 2007) y en el listado de flora cantábrica de interés (Llamas et al. 2007).

\section{Orchis provincialis $\mathrm{L}$.}

ESPAÑA: Palencia: Aguilar de Campoo, Cordovilla de Aguilar, 30TUM9948, $1016 \mathrm{~m}$, pastizal de diente, 11-V-2010, A. Ruiz de Gopegui \& L. García, LEB 104040.

Aportamos una nueva localidad septentrional a añadir a la que se dio a conocer recientemente en las Loras 
palentinas (Ruiz de Gopegui et al. 2010: 154). Su rareza en la provincia y en el conjunto de la comunidad autónoma aconsejó su inclusión en el catálogo de flora protegida de Castilla y León (Anon. 2007), así como en el listado de flora cantábrica de interés (Llamas et al. 2007).

\section{*Orchis spitzelii Saut. ex W. D. J. Koch}

ESPAÑA: Palencia: Triollo, La Lastra, 30TUn6250, $1330 \mathrm{~m}$, matorrales de sabina rastrera con gayuba en sustrato calizo, 25-V-2011, P. Bariego, Fig. 4; Ibidem, 09-VI-2011, E. Álvarez, SALA 142387; Velilla del Río Carrión, Camporredondo de Alba, 30TUN6149, $1410 \mathrm{~m}$, pastizal calizo entre gayubas y sabina rastrera, 09-VI-2011, E. Álvarez, SALA 142388.

Novedad provincial destacada, a tenor de su rareza en la Península Ibérica, donde sólo se conoce en las provincias de Lérida y León (Aedo 2005: 139; Del Egido \& Puente 2008). En la provincia de Palencia se han localizado un par de poblaciones cercanas entre sí y no muy distantes de la localidad leonesa que confirmó su presencia en la Cordillera Cantábrica (Del Egido \& Puente 2008). En este trabajo y en la monografía de Flora iberica, ya se indicaba la estrecha relación de este taxón con 0 . cazorlensis Lacaita, de la que se diferencia por tener un espolón relativamente más largo en relación con el tamaño del labelo, aunque también señalan ciertas dificultades para la correcta identificación del material ibérico. En este sentido, hemos identificado las plantas palentinas con 0 . spitzelii basándonos en la longitud relativamente larga de los espolones y comprobando la existencia de diferencias en los tamaños, disposición y coloración de las piezas florales con materiales y fotografías del taxon de Lacaita, aunque sin descartar la posibilidad de que una nueva visión de conjunto de las poblaciones ibéricas de ambas especies puede contribuir a aclarar la identidad de cada taxón, sus rangos de variabilidad y sus áreas de distribución. En cualquier caso, estas poblaciones palentinas y leonesas son muy interesantes desde el punto de vista biogeográfico por lo que requieren una especial atención en las iniciativas de conservación de flora que puedan plantearse en adelante. No en vano, está incluida en la Lista Roja Española 2008 (Moreno 2008: 53) bajo la categoría en Peligro Crítico (Fig. 4).

\section{Platanthera bifolia (L.) Rich.}

ESPAÑA: Palencia: Brañosera, Salcedillo, 30TUN9755, 1230 $\mathrm{m}$, prados higroturbosos, 28-VI-2010, A. Ruiz de Gopegui, LEB 105767; Brañosera, Salcedillo, 30TUN9455, 1330 m, brezal higrófi-

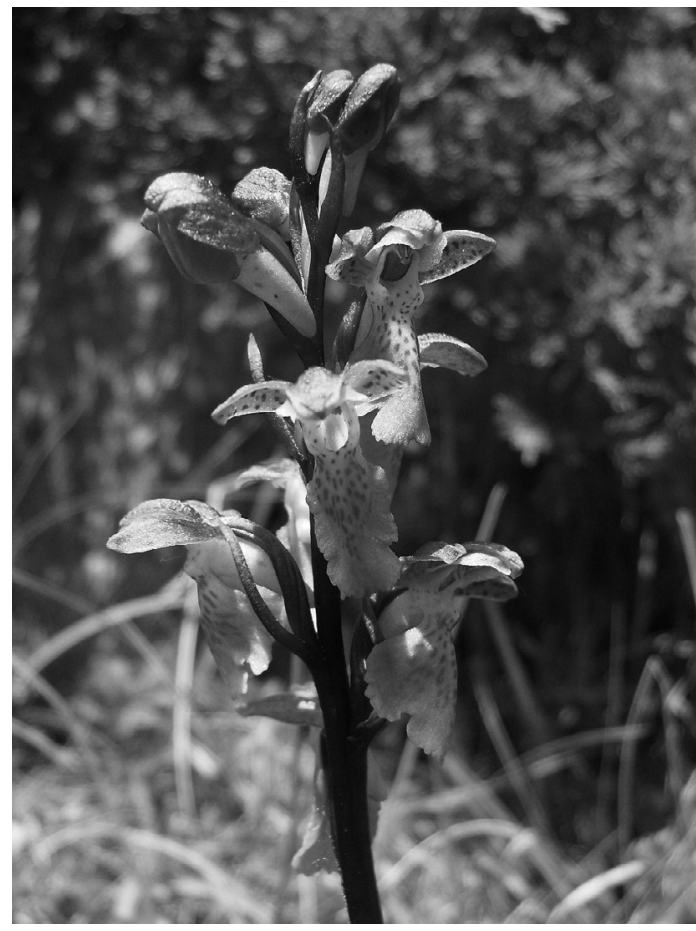

Fig. 4- Orchis spitzelii Saut. ex W. D. J. Koch.

10, 3-VII-2010, A. Rodríguez, LEB 104631; Aguilar de Campoo, Monte Royal, 30TUN925381, 950 m, 6-VI-2011, A. Ruiz de Gopegui \& L. García, LEB 105766; San Cebrián de Mudá, San Martín de Perapertú, 30TUN8850, 1160 m, pastizal, 28-V-2011, E. Álvarez, M. Velez \& J. Cuesta, Cervera de Pisuerga, Quintanaluengos, Laguna de Sosa, 30TUN8344, $960 \mathrm{~m}$, pastizal meso-higrófilo ácido, 10-VI-2011, A. Rodriguez, LEB 105941.

Nuevas localidades a añadir a las dos menciones previas de la Montaña Palentina (Aedo et al. 1994: 94). Aunque nunca en poblaciones de gran tamaño, aparece dispersa en el tercio norte provincial. Incluida en el listado de flora cantábrica de interés (Llamas et al. 2007).

\section{*Serapias cordigera L.}

ESPAÑA: PALENCIA: Sotobañado y Priorato, 30TUN8016, 913 m, brezal, 31-V-2011, A. Ruiz de Gopegui, LEB 105391.

Primera cita palentina de esta orquídea que parece ser muy rara en el contexto provincial, a juzgar por esta única localidad conocida, a diferencia de lo que sucede en el cercano norte de la provincia de Burgos donde parece estar relativamente extendida (Alejandre et al. 2006: 833). 


\section{*Serapias parviflora L.}

ESPAÑA: Palencia: Aguilar de Campoo, Pozancos, 30TUN9828, $1000 \mathrm{~m}$, pastizal de diente, 10-VI-2010, A. Ruiz de Gopegui \& L. García, LEB 104045; Aguilar de Campoo, Valdegama, 30TVN0232, 890 m, pastizal meso-higrófilo ácido, 13-V-2010, A. Rodríguez, LEB 104655; Cervera de Pisuerga, Perazancas de Ojeda, La Bárcena, 30TVN8436, $1010 \mathrm{~m}$, pastos en suelos margosos básicos rezumantes, 11-VI-2011, P. Bariego, PB3650, LEB 105641.

Novedad provincial que se restringe al norte de la provincia, donde crece en pastos higrófilos, tanto acidófilos como basófilos. Al igual que la anterior, también parece ser una planta algo más frecuente en la vecina provincia burgalesa (Alejandre et al. 2006: 834).

\section{Spiranthes spiralis (L.) Chevall.}

ESPAÑA: Palencia: Cervera de Pisuerga, Rabanal de los Caballeros, 30TUN7949, $1050 \mathrm{~m}$, ladera pedregosa caliza, 9-IX-2004, A. Ruiz de Gopegui, Aguilar de Campoo, Embalse de Aguilar, 30TVN9239, $950 \mathrm{~m}$, claros de pasto en brezal en borde de pinar, 28IX-2009, A. Rodríguez, LEB104177; Cervera de Pisuerga, Rabanal de los Caballeros, 30TUN7851, 1150 m, camino pisoteado, 3-IX-2011, A. Ruiz de Gopegui, RdeG 588; Cervera de Pisuerga, Rabanal de los Caballeros, 30TUN7949, 1050 m, ladera pedregosa caliza, 1-IX-2011, A. Ruiz de Gopegui, RdeG 589; Aguilar de Campoo, Lomilla,
30TUN9336, $900 \mathrm{~m}$, pradera con brezal, 5-IX-2011, A. Rodríguez, LEB 105913; Salinas de Pisuerga, 30TUN8646, $1110 \mathrm{~m}$, pastizal entre robles, 04-IX-2011, E. Álvarez; San Cebrián de Mudá, San Martín de Perapertú, 30TUN8851, 1140 m, pastizal calizo, 23-IX-2011, A. Ruiz de Gopegui, RdeG 591.

Salvo la indicación provincial entre paréntesis de Patallo \& Aedo (2005: 70), probablemente basada en la indicación de Leroy \& Lainz (1954: 123) sobre su presencia en la vega de Carrión de los Condes, no se conocen otras citas para este taxón en la provincia de Palencia. Aportamos ahora un buen número de localidades del norte provincial, donde crece en pastos más o menos secos tanto en sustratos calizos como en silíceos. Quizás su floración tardía pueda ser la causa de la inexistencia de citas previas ya que no parece ser una planta rara en el norte provincial.

\section{AGRADECIMIENTOS}

Agradecemos la ayuda y colaboración prestada en el trabajo de campo a Yolanda Ruiz y Laurentino García Cayón, miembros del grupo GEMPA, así como a Sonia Oreca y a Javier Cuesta. Los comentarios de Carlos Aedo y Javier Benito nos han ayudado con la identificación de $O$. spitzelii. También queremos dar las gracias a Luis Delgado y María Santos por cedernos algunos interesantes datos de sus herborizaciones del Cerrato y los páramos de Astudillo-Torquemada.

\section{BIBLIOGRAFIA}

Aedo, C. 2005. Orchis L. En C. Aedo \& A. Herrero (Eds.), Flora iberica 21: 114-146. Madrid.

Aedo, C.; Aldasoro, J. J.; Argüelles, J. M.; Carlón, L.; Díez Riol, A.; González Del Valle, J. M.; Laínz, M.; Moreno Moral, G.; Patallo, J. \& Sánchez Pedraja, O. 2000. Contribuciones al conocimiento de la flora cantábrica, IV. Bol. Cien. Nat. R.I.D.E.A. 46: 7-119.

Aedo, C.; Aldasoro, J.J.; Argüelles, J.M.; Carlón, L.; Díez Riol, A.; Gómez CASARes, G.; González Del Valde, J.M.; Gullén Oterino, A.; Laínz, M.; Moreno Moral, G.; Patallo, J. \& SÁnchez PedRAJA, 0. 2001. Contribuciones al conocimiento de la flora cantábrica, V. Bol. Cien. Nat. R.I.D.E.A. 47: 7-52.

Aedo, C.; Aldasoro, J. J.; Argüelles, J. M.; Díaz Alonso, J. L.; Díez Riol, A.; González Del Valle, J. M.; Laínz, M.; Moreno Moral, G.; Patallo, J. \& Sánchez Pedraja, 0. 1994. Contribuciones al conocimiento de la flora cantábrica, II, Fontqueria 40: 67-100.

Aedo, C.; Aldasoro, J. J.; Argüelles, J. M.; Díaz Alonso, J. L.; González Del Valle, J. M.; HerRÁ, C.; LAínZ, M.; Moreno Moral, G.; Patallo, J. \& Sánchez Pedraja, O. 1993. Contribuciones al conocimiento de la flora cantábrica. Fontqueria 36: 349-374.
Alarcón, M. L. \& Aedo, C. 2002. Revisión taxonómica del género Cephalanthera (Orchidaceae) en la Península Ibérica e Islas Baleares. Anales Jard. Bot. Madrid 59 (2): 227-248.

Alarcón, M. L. \& Aedo, C. 2005. Cephalanthera Rich. En C. Aedo \& A. Herrero (Eds.), Flora iberica 21: 54-58. Madrid. Aldasoro, J. J. \& SÁez, L. 2005. Ophrys L. En C. Aedo \& A. Herrero (Eds.), Flora iberica 21: 165-195. Madrid.

Alejandre SÁenz, J. A.; García López, J. M. \& Mateo Sanz, G. (Eds.). 2006. Atlas de la flora vascular silvestre de Burgos. Junta de Castilla y León-Caja Rural Burgos. Burgos.

Anon. 2007. Decreto 63/2007, de 14 de junio, por el que se crean el Catálogo de Flora Protegida de Castilla y León y la figura de protección denominada Microrreserva de Flora. Boletín Oficial de Castilla y León 119: 13197-13204. Valladolid.

Argüelles, J. M.; Carlón, L.; Gómez Casares, G.; González del Valle, J. M.; Laínz, M.; Moreno Moral, G. \& SÁnchez Pedraja, Ó. 2005. Contribuciones al conocimiento de la flora cantábrica, VII. Bol. Cien. Nat. R.I.D.E.A. 49: 147-194

Bariego, P. \& Gastón, A. 2005. Aproximación al catálogo de las orquídeas silvestres de la provincia de Zamora. Ecología 19: 149-164. 
Benito ayuso, J. \& Tabuenca marraco, J. M. 2000. Apuntes sobre orquídeas (principalmente del Sistema Ibérico). Est. Mus. Cienc. Nat. Álava 15: 103-126.

Benito Ayuso, J. \& Hermosilla, C. 1998. Dos nuevas especies ibéricas, Epipactis cardina y Epipactis hispanica, más alguno de sus híbridos: Epipactis x conquensis (E. cardina $\mathrm{x} E$. parviflora) y Epipactis x populetorum (Epipactis helleborine $x$ E. hispanica). Estud. Mus. Ci. Nat. Álava 13: 103-115.

CRespo, M. B. 2005. Epipactis Zinn. En C. Aedo \& A. Herrero (Eds.), Flora iberica 21: 22-54. Madrid.

Del Egido, F. \& Puente, E. 2008. Orchis spitzelii Saut. ex W. D. J. Koch en la Cordillera Cantábrica. Flora montiberica 38: 13-15.

Gandoguer, M. 1898. Notes sur la flore espagnole, III. Mon sixième voyage dans la Péninsule Ibérique en 1898. Bull. Soc. Bot. France 45: 588-604.

Leroy, E. \& Laínz, M. 1954. Contribución al catálogo de la flora palentina. Collect. Bot. (Barcelona) 4: 81-123.
Llamas, F.; Acedo, C.; Lence, C.; Alonso, R.; Molina; A. \& CASTRO, V. 2007. Flora Cantábrica de interés en Castilla y León. Naturalia Cantabricae 3: 57-78.

LÓPEZ, N. 2005. Neotinea Rchb. En C. Aedo \& A. Herrero (Eds.), Flora iberica 21: 111-114. Madrid.

Moreno, J. C. (coord.). 2008. Lista Roja 2008 de la flora vascular española. Dirección General de Medio Natural y Política Forestal (Ministerio de Medio Ambiente, y Medio Rural y Marino) - SEBCP. Madrid.

Oria de Rueda, J. A.; Díez, J. \& Rodríguez, M. 1996. Guía de las plantas silvestres de Palencia. Ed. Cálamo, Palencia.

Patallo, J. \& Aedo, C. 2005. Spiranthes Rich. En C. Aedo \& A. Herrero (Eds.), Flora iberica 21: 69-72. Madrid.

Ruiz De Gopegui, J. A; De Paz, E.; Alonso R.; Ferreras, N.; García, R. M.; Alonso, S.; Rodríguez, A. \& García. M. E. 2010. Aportaciones al conocimiento de la orquidoflora palentina. Acta Bot. Malacitana 35: 152-155. 